\title{
A Consistent Dissipative Time Integration Scheme for Structural Dynamics. Application to Rotordynamics.
}

\author{
Ludovic Noels, Laurent Stainier†, and Jean-Philippe Ponthot ${ }^{\ddagger}$ \\ Université de Liège, B-4000 Liège, Belgium
}

\begin{abstract}
Recently, robust implicit energy and momentum conserving algorithms have been developed in the non-linear range. In previous works, the authors extended these algorithms to hypoelasticity-based constitutive models. In this paper we introduce numerical dissipation, opening the way to more complex simulations such as blade-loss in a turbofan.
\end{abstract}

\section{Introduction}

In order to integrate the equations of evolution of dynamical systems, one can resort to two families of algorithms: the implicit family and the explicit family. In this paper, we focus on the implicit family. The most widely used implicit algorithm is the Newmark algorithm. ${ }^{1}$ For linear models, this algorithm is unconditionally stable if some conditions on the parameters are verified. For non-linear models, Belytschko and Schoeberle, ${ }^{2}$ and Hughes ${ }^{3}$ proved that the discrete energy is bounded if it remains positive. Nevertheless, Hughes et al. ${ }^{4,5}$ have proved that, in the non-linear range, the Newmark algorithm remains physically consistent only for small time step sizes. The contradiction between these two observations results from the fact that the discrete energy is computed from the work of the internal forces, which is different from the internal potential when the Newmark algorithm is used in the non-linear range. To avoid divergence due to numerical instabilities, numerical damping was introduced, leading to the generalized- $\alpha$ methods. ${ }^{6}$ Nevertheless, the unconditional stability of these methods occurs only for linear systems or asymptotically for the high frequency in the non-linear range. ${ }^{7}$

Therefore a new kind of dynamics integration algorithms has appeared that verifies the mechanical laws of conservation (i.e. conservation of linear momentum, angular momentum and total energy) and that remains stable in the nonlinear range. The first algorithm (EMCA or Energy Momentum Conserving Algorithm) verifying these properties was described by Simo and Tarnow. ${ }^{8}$ It consists in a mid-point scheme with an adequate evaluation of the internal forces. This adequate evaluation (i.e. that leads to a consistent algorithm) was given by Simo and Tarnow ${ }^{8}$ for a Saint Venant-Kirchhoff hyperelastic material. A generalization to other hyperelastic models was given by Gonzalez. ${ }^{9}$ The extension

\footnotetext{
*Research Fellow FNRS, Dept. of Aerospace, Mechanics and Materials, LTAS-MCT, 1 chemin des chevreuils.

${ }^{\dagger}$ Research Associate FNRS, Dept. of Aerospace, Mechanics and Materials, LTAS-MCT, 1 chemin des chevreuils, Member AIAA.

${ }^{\ddagger}$ Senior Research Associate, Dept. of Aerospace, Mechanics and Materials, LTAS-MCT, 1 chemin des chevreuils.
} 
of EMCA to hyperelastic constitutive models was recently extended to dynamic finite deformation plasticity by Meng and Laursen. ${ }^{10}$ In such a formulation, the algorithm remains energy conserving when no plastic deformation occurs, and "dissipates energy in a manner consistent with the physical model in use" (sic) when plastic deformation occurs. In a previous work, ${ }^{11,12}$ we have developed a new expression of the internal forces, ensuring the conservation laws of the mechanics for a hypoelastic constitutive model. Besides, Armero and Petöcz ${ }^{13,14}$ proposed a consistent treatment of contact interactions in the non-linear range.

To avoid the lack of convergence due to the presence of high frequency modes, numerical dissipation was introduced in the conserving algorithms (for hyperelastic materials only) by Armero and Romero. ${ }^{15,16}$ This numerical scheme (EDMC or Energy Dissipative Momentum Conserving) was proved to be dissipative in the non-linear range. In this paper, we introduce this numerical formulation in our algorithm for the hypoelastic model. Thanks to this numerical dissipation we are able to simulate complex dynamic events such as blade-loss in a turbo-engine.

\section{The EMCA Method}

For an integration from time $t^{n}$ to time $t^{n}+\Delta t=t^{n+1}$, the relations between positions $(\vec{x})$, velocities $(\dot{\vec{x}})$ and accelerations $(\ddot{\vec{x}})$ are given by

$$
\begin{aligned}
& \vec{x}^{n+\frac{1}{2}}=\frac{\vec{x}^{n+1}+\vec{x}^{n}}{2} \\
& \dot{\vec{x}}^{n+\frac{1}{2}}=\frac{\vec{x}^{n+1}-\vec{x}^{n}}{\Delta t}=\frac{\dot{\vec{x}}^{n+1}+\dot{\vec{x}}^{n}}{2} \\
& \ddot{\vec{x}}^{n+\frac{1}{2}}=\frac{\dot{\vec{x}}^{n+1}-\dot{\vec{x}}^{n}}{\Delta t}=\frac{\ddot{\vec{x}}^{n+1}+\ddot{\vec{x}}^{n}}{2}
\end{aligned}
$$

After finite element discretization, the balance law for node $\xi$ is given by

$$
M^{\xi \mu}\left[\ddot{\vec{x}}^{n+\frac{1}{2}}\right]^{\mu}=\left[\vec{F}_{\text {ext }}^{n+\frac{1}{2}}-\vec{F}_{\text {int }}^{n+\frac{1}{2}}\right]^{\xi}
$$

where $M^{\xi \mu}$ is the mass matrix, and $\vec{F}_{i n t}^{n+\frac{1}{2}}\left(x^{n}, x^{n+1}\right)$ is the expression of the internal forces in configuration $n+\frac{1}{2}$.

In a previous work, ${ }^{11}$ we have developed a new expression of the internal forces, ensuring the conservation laws of the mechanics for a hypoelastic constitutive model:

$$
\begin{aligned}
{\left[\vec{F}_{\text {int }}^{n+\frac{1}{2}}\right]^{\xi} } & =\frac{1}{2}\left[\vec{F}_{i n t}^{*}+\vec{F}_{i n t}^{* *}\right]^{\xi} \text { with } \\
{\left[\vec{F}_{i n t}^{*}\right]^{\xi} } & =\frac{1}{2} \int_{V_{0}}\left\{\left[\mathbf{I}+\mathbf{F}_{n}^{n+1}\right]\left[\boldsymbol{\Sigma}^{n}+\mathbf{C}^{*}\right] \mathbf{f}_{0}^{n T} \vec{D}^{\xi} J_{0}^{n}\right\} d V_{0} \\
{\left[\vec{F}_{i n t}^{* *}\right]^{\xi} } & =\frac{1}{2} \int_{V_{0}}\left\{\left[\mathbf{I}+\mathbf{f}_{n}^{n+1}\right]\left[\boldsymbol{\Sigma}^{n+1}+\mathbf{C}^{* *}\right] \mathbf{f}_{0}^{n+1} \vec{D}^{\xi} J_{0}^{n+1}\right\} d V_{0}
\end{aligned}
$$

In this expression, the gradient of deformation (two point tensor) $\mathbf{F}$ between configurations $m$ and $n$ is defined by

$$
\mathbf{F}_{m}^{n}=\frac{\partial \vec{x}^{n}}{\partial \vec{x}^{m}}
$$

The tensor $\mathbf{f}$ represents $\mathbf{F}^{-1}$. The determinant of $\mathbf{F}_{m}^{n}$ is denoted by the scalar 
$J_{m}^{n}$. The Green-Lagrange strain tensor $\mathbf{G L}_{m}^{n}$, the Almansi strain tensor $\mathbf{A}_{m}^{n}$ and the natural strain tensor $\mathbf{E}_{m}^{n}$ are defined as

$$
\begin{aligned}
\mathbf{G L}_{m}^{n} & =\frac{1}{2}\left[\mathbf{F}_{m}^{n T} \mathbf{F}_{m}^{n}-\mathbf{I}\right] \\
\mathbf{A}_{m}^{n} & =\frac{1}{2}\left[\mathbf{I}-\mathbf{f}_{m}^{n T} \mathbf{f}_{m}^{n}\right] \\
\mathbf{E}_{m}^{n} & =\frac{1}{2} \ln \left[\mathbf{F}_{m}^{n T} \mathbf{F}_{m}^{n}\right]
\end{aligned}
$$

The Cauchy stress tensor is evaluated in the configuration $n$ and is refered to as $\boldsymbol{\Sigma}^{n}$. Finally, $\vec{D}$ denotes the derivatives of shape functions (in the reference configuration). For hypoelastic constitutive laws, the Cauchy stress tensor is computed from a stress increment $\boldsymbol{\Delta} \boldsymbol{\Sigma}_{n}^{n+1}$ between two successive configurations deduced from the natural strain tensor

$$
\Delta \boldsymbol{\Sigma}_{n}^{n+1}=\mathcal{H}: \mathbf{E}_{n}^{n+1}
$$

with $\mathcal{H}$ the Hooke fourth order tensor. The final rotation scheme ${ }^{17,18}$ is defined by the following relation ( $\mathbf{R}$ is the rotation tensor of the gradient deformation F)

$$
\boldsymbol{\Sigma}^{n+1}=\mathbf{R}_{n}^{n+1}\left[\boldsymbol{\Sigma}^{n}+\Delta \boldsymbol{\Sigma}_{n}^{n+1}-\mathbf{s}^{\mathbf{c}}\right] \mathbf{R}_{n}^{n+1^{T}}
$$

If plastic deformations occur, $\mathbf{s}^{\mathbf{c}}$ is the purely deviatoric correction tensor resulting from the radial return mapping ( $J 2$ plasticity). ${ }^{18-20}$ We have demonstrated $^{11}$ that expression (5) preserves the linear momentum (thanks to the fact that $\sum_{\xi} \vec{D}^{\xi}=0$ ), preserves the angular momentum (if $\boldsymbol{\Sigma}, \mathbf{C}^{*}$ and $\mathbf{C}^{* *}$ are symmetric) and that the dissipation of the internal forces work is given by

$$
\begin{aligned}
& \Delta_{i n t}=\frac{1}{2} \int_{V^{0}}\left\{\mathbf{G}^{\mathbf{p l}^{n+1}}{ }_{n}: \boldsymbol{\Sigma}^{n} J_{0}^{n}\right\} d V_{0}+\frac{1}{2} \int_{V^{0}}\left\{\mathbf{G L}_{n}^{n+1}: \mathbf{C}^{*} J_{0}^{n}\right\} d V_{0} \\
& +\frac{1}{2} \int_{V^{0}}\left\{\mathbf{A}_{n}^{\mathbf{p l}^{n+1}}: \boldsymbol{\Sigma}^{n+1} J_{0}^{n+1}\right\} d V_{0}+\frac{1}{2} \int_{V^{0}}\left\{\mathbf{A}_{n}^{n+1}: \mathbf{C}^{* *} J_{0}^{n+1}\right\} d V_{0}
\end{aligned}
$$

where $\mathbf{G L}^{\mathbf{p l}}$ and $\mathbf{A}^{\mathbf{p l}}$ are the plastic part of $\mathbf{G} \mathbf{L}$ and $\mathbf{A}$, respectively, computed from $\mathbf{s}^{\mathbf{c}} .{ }^{11,12}$ These parts are computed from the following method. If $\mathbf{E}^{\mathrm{el}}$ is the elastic part of the natural deformations

$$
\mathcal{H}: \mathbf{E}_{n}{ }^{\mathbf{e}}{ }^{n+1} \equiv \mathcal{H}: \mathbf{E}_{n}^{n+1}-\mathbf{s}^{c}
$$

Tensor $\mathbf{U}_{n}^{\mathbf{e}}{ }_{n}^{n+1}$ is defined by

$$
\mathbf{E}_{n}^{\mathbf{e}}{ }_{n}^{n+1} \equiv \frac{1}{2} \ln \left[\mathbf{U}_{n}^{\mathbf{e l}^{n+1}} \mathbf{U}_{n}^{\mathbf{e l}}{ }_{n}^{n+1}\right]
$$

The existence of $\mathbf{U}^{\mathrm{el}}{ }_{n}^{n+1}$ is ensured by the symmetric nature of $\mathbf{E}^{\mathrm{el}}{ }_{n}^{n+1}$. Therefore elastic tensors $\mathbf{G} \mathbf{L}_{n}{ }^{\mathbf{e} n+1}$ and $\mathbf{A}{ }_{n}^{\mathbf{e l}}{ }_{n}^{n+1}$ are defined from $\mathbf{U}_{n}^{\mathbf{e l}}{ }_{n}^{n+1}$

$$
\begin{aligned}
& \mathbf{G L}^{\mathbf{e}^{\mathbf{e}^{n+1}}} \equiv \frac{1}{2}\left[\mathbf{U}_{n}^{\mathbf{e}{ }_{n}^{n+1}} \mathbf{U}_{n}^{\mathrm{el}}{ }_{n}^{n+1}-\mathbf{I}\right] \\
& \mathbf{A}_{n}^{\mathbf{e l}}{ }_{n}^{n+1} \equiv \frac{1}{2} \mathbf{R}_{n}^{n+1}\left[\mathbf{I}-\mathbf{U}_{n}^{\mathbf{e l}^{n+1}-1} \mathbf{U}_{n}^{\mathbf{e}{ }^{n+1}}\right] \mathbf{R}_{n}^{n+1^{T}}
\end{aligned}
$$

The elastic part $\mathbf{G L}^{\mathrm{el}}$ and $\mathbf{A}^{\mathrm{el}}$ of, respectively, GL and $\mathbf{A}$ contribute to an increase of the reversible energy (i.e. that is stored as internal energy and could 
be released by the material $\left.{ }^{11}\right)$. Therefore, the plastic part $\mathbf{G L}^{\mathbf{p l}}$ and $\mathbf{A}^{\mathbf{p l}}$ of, respectively, GL and $\mathbf{A}$ are obtained from

$$
\begin{gathered}
\mathbf{G L}_{n}^{\mathbf{p l}{ }_{n}^{n+1}} \equiv \mathbf{G L}_{n}^{n+1}-\mathbf{G L}_{n}^{\mathbf{e l}}{ }_{n+1}^{n+1} \\
\mathbf{A}_{n}^{\mathbf{p l}{ }_{n}^{n+1}} \equiv \mathbf{A}_{n}^{n+1}-\mathbf{A}_{n}^{\mathbf{e l}{ }_{n}^{n+1}}
\end{gathered}
$$

To be physically consistent, relation (12) has to be related to a physical quantity. The positive internal plastic dissipation can be expressed from a volumic dissipation $D_{\text {int }}$ obtained from ${ }^{12}$

$$
D_{i n t} \simeq \frac{1}{2} \varepsilon_{n}^{p n+1}\left[\Sigma_{v}{ }^{n+1}+\bar{\alpha}^{n+1}\right] J_{0}^{n+1}+\frac{1}{2} \varepsilon_{n}^{p n+1}\left[\Sigma_{v}{ }^{n}+\bar{\alpha}^{n}\right] J_{0}^{n}
$$

where $\varepsilon^{p}$ is the equivalent plastic strain, $\Sigma_{v}$ is the von Mises yield stress and $\bar{\alpha}$ is the equivalent back-stress. The internal plastic dissipation is expressed by

$$
\Delta_{\text {int }}^{p h y}=\int_{V_{0}}\left\{D_{i n t}\right\} d V_{0}>0
$$

From there, we can deduce the tensors $\mathbf{C}^{*}$ and $\mathbf{C}^{* *}$ that lead to an energy conserving scheme ${ }^{12}$

$$
\begin{aligned}
\mathbf{C}^{*} & =\frac{\frac{D_{\text {int }}}{J_{0}^{n}}-\boldsymbol{\Sigma}^{n}: \mathbf{G L}^{\mathbf{p l}}{ }_{n}^{n+1}}{\mathbf{G L}_{n}^{n+1}: \mathbf{G L}_{n}^{n+1}} \mathbf{G L}_{n}^{n+1} \\
\mathbf{C}^{* *} & =\frac{\frac{D_{\text {int }}}{J_{0}^{n+1}}-\boldsymbol{\Sigma}^{n+1}: \mathbf{A}_{n}^{\mathbf{p l}^{n+1}}}{\mathbf{A}_{n}^{n+1}: \mathbf{A}_{n}^{n+1}} \mathbf{A}_{n}^{n+1}
\end{aligned}
$$

These tensors are symmetric (leading to the conservation of the angular momentum) and are equal to zero when no plastic deformation occurs, as assumed in the beginning. If there are plastic deformations, the dissipation $\Delta_{\text {int }}$ is then equal to $\Delta_{\text {int }}^{\text {phy }}>0$.

\section{The EDMC Scheme}

To avoid the lack of convergence due to the presence of high frequency modes, numerical dissipation was introduced in the conserving algorithms (for hyperelastic materials only) by Armero and Romero. ${ }^{15,16}$ A dissipation velocity $\vec{G}_{\text {diss }}$ is introduced in relations (2) and (3) and a dissipation force $\vec{F}_{\text {diss }}$ is introduced in (4), leading to

$$
\begin{aligned}
& {\left[\vec{x}^{n+1}\right]^{\xi}=\left[\vec{x}^{n}\right]^{\xi}+\Delta t\left[\frac{\dot{\vec{x}}^{n+1}+\dot{\vec{x}}^{n}}{2}+\vec{G}_{\text {diss }}^{n+\frac{1}{2}}\right]^{\xi}} \\
& {\left[\dot{\vec{x}}^{n+1}\right]^{\xi}=\left[\dot{\vec{x}}^{m}\right]^{\xi}+\frac{\Delta t}{2}\left[\ddot{\vec{x}}^{n+1}+\ddot{\vec{x}}^{n}\right]^{\xi}}
\end{aligned}
$$

and to

$$
M^{\xi \mu}\left[\frac{\ddot{\vec{x}}^{n+1}+\ddot{\vec{x}}^{n}}{2}\right]^{\mu}=\left[\vec{F}_{\text {ext }}^{n+\frac{1}{2}}-\vec{F}_{\text {int }}^{n+\frac{1}{2}}\right]^{\xi}-\left[\vec{F}_{\text {diss }}^{n+\frac{1}{2}}\right]^{\xi}
$$

The simultaneous presence of dissipation velocities and forces is the necessary condition to avoid a bifurcation in spectral analysis. Let $D_{K} \geq 0$ be a specific potential of the kinetic dissipation. Armero and Romero ${ }^{16}$ proposed the 
following expression for the dissipation velocities

$$
M^{\xi \mu}\left[\vec{G}_{d i s s}^{n+\frac{1}{2}}\right]^{\mu}=\int_{V_{0}}\left\{\rho_{0} \varphi^{\xi} D_{K} \frac{\dot{\vec{x}}^{n+1}+\dot{\vec{x}}^{n}}{\left\|\dot{\vec{x}}^{n+1}\right\|^{2}-\left\|\dot{\vec{x}}^{n}\right\|^{2}}\right\} d V_{0}
$$

where

$$
\dot{\vec{x}}=\varphi^{\xi}[\dot{\vec{x}}]^{\xi}
$$

This expression leads to an angular momentum conserving algorithm, since ${ }^{16}$

$$
\left[\dot{\vec{x}}^{n+1}-\dot{\vec{x}}^{n}\right]^{\xi} \wedge M^{\xi \mu}\left[\vec{G}_{\text {diss }}^{n+\frac{1}{2}}\right]^{\mu}=0
$$

and the kinematic dissipation $\Delta_{K}$ is computed from ${ }^{15,16}$

$$
\Delta_{K}=\left[\dot{\vec{x}}^{n+1}-\dot{\vec{x}}^{n}\right]^{\xi} \cdot M^{\xi \mu}\left[\vec{G}_{d i s s}^{n+\frac{1}{2}}\right]^{\mu}=\int_{V_{0}}\left\{\rho_{0} D_{K}\right\} d V_{0} \geq 0
$$

Conservation of the linear momentum does not depends on the dissipation velocity. ${ }^{15,16}$ If we want a first order dissipative scheme, we can choose $D_{K}$ such as

$$
D_{K}=\chi \frac{1}{2}\left[\left\|\dot{\vec{x}}^{n+1}\right\|-\left\|\dot{\vec{x}}^{n}\right\|\right]^{2} \geq 0
$$

where $\chi$ is a parameter that controls the numerical dissipation. Since expression (30) is of the second order in $\Delta t$, the expression of the dissipation velocity (26) is of the first order in $\Delta t$, leading to a first order scheme. Let us note that, contrarily to the form proposed by Armero and Romero ${ }^{16}$ this expression leads to an absence of dissipation for a uniform rotation. Relations (26) and (30) could be approximated (if the consistent mass matrix is replaced by the lumped mass matrix in (26)) by (no sum on $\xi$ )

$$
\left[\vec{G}_{\text {diss }}^{n+\frac{1}{2}}\right]^{\xi}=\chi \frac{\left\|\left[\dot{\vec{x}}^{n+1}\right]^{\xi}\right\|-\left\|\left[\dot{\vec{x}}^{n}\right]^{\xi}\right\|}{\left\|\left[\dot{\vec{x}}^{n+1}\right]^{\xi}\right\|+\left\|\left[\dot{\vec{x}}^{n}\right]^{\xi}\right\|} \frac{\left[\dot{\vec{x}}^{n+1}+\dot{\vec{x}}^{n}\right]^{\xi}}{2}
$$

Armero and Romero ${ }^{16}$ established an expression of the dissipation forces for hyperelastic materials. In this paper we propose, for the hypoelastic model, to introduce numerical dissipation in a similar way. By analogy with the relations (5) and (12), we propose the dissipation forces ${ }^{21}$

$$
\begin{aligned}
{\left[\vec{F}_{\text {diss }}^{n+\frac{1}{2}}\right]^{\xi}=\frac{1}{4} \int_{V_{0}}\{[\mathbf{I}+} & \left.\left.\mathbf{F}_{n}^{n+1}\right] \mathbf{D}^{*} \mathbf{f}_{0}^{n T} \vec{D}^{\xi} J_{0}^{n}\right\} d V_{0} \\
& +\frac{1}{4} \int_{V_{0}}\left\{\left[\mathbf{I}+\mathbf{f}_{n}^{n+1}\right] \mathbf{D}^{* *} \mathbf{f}_{0}^{n+1^{T}} \vec{D}^{\xi} J_{0}^{n+1}\right\} d V_{0}
\end{aligned}
$$

which leads to a numerical internal dissipation

$$
\Delta_{W}=\frac{1}{2} \int_{V_{0}}\left\{\mathbf{G L}_{n}^{n+1}: \mathbf{D}^{*} J_{0}^{n}\right\} d V_{0}+\frac{1}{2} \int_{V_{0}}\left\{\mathbf{A}_{n}^{n+1}: \mathbf{D}^{* *} J_{0}^{n+1}\right\} d V_{0}
$$


Therefore, tensors $\mathbf{D}^{*}$ and $\mathbf{D}^{* *}$ are taken equal to

$$
\begin{aligned}
\mathbf{D}^{*} & =\frac{\frac{D_{W}}{J_{0}^{n}}}{\mathbf{G L}_{n}^{n+1}: \mathbf{G L}_{n}^{n+1}} \mathbf{G L}_{n}^{n+1} \\
\mathbf{D}^{* *} & =\frac{\frac{D_{W}}{J_{0}^{n+1}}}{\mathbf{A}_{n}^{n+1}: \mathbf{A}_{n}^{n+1}} \mathbf{A}_{n}^{n+1}
\end{aligned}
$$

with $D_{W} \geq 0$ defined such that

$$
\Delta_{W}=\int_{V_{0}}\left\{D_{W}\right\} d V_{0}>0
$$

Let us then define

$$
D_{W}=\frac{\chi}{2} \mathbf{E}_{n}^{\mathbf{e l}^{n+1}}: \mathcal{H}: \mathbf{E}_{n}^{\mathrm{el}^{n+1}} J_{0}^{n} \geq 0
$$

where $\mathbf{E}^{\mathrm{el}}$ is the elastic part of $\mathbf{E}$ computed from (14). Since expression (37) is of the second order in $\Delta t$, expression (33) is of the first order in $\Delta t$ leading to a first order scheme. Moreover, since $\sum_{\xi} \vec{D}=0$, expression (33) leads to a linear momentum conserving scheme. By analogy with (5), since $\mathbf{D}^{*}$ and $\mathbf{D}^{* *}$ are symmetric, the angular momentum is also conserved. The total numerical dissipation is

$$
\Delta_{n u m}=\Delta_{K}+\Delta_{W} \geq 0
$$

Now, we will prove that the potentials (30) and (37) lead to a spectral analysis without bifurcation. Let us take the hypothesis of small transformations, without plasticity:

$$
\begin{aligned}
J_{0}^{n+1} & \simeq J_{0}^{n} \\
\mathbf{G L}^{\mathbf{e l}^{n+1}} & \simeq \varepsilon^{\mathbf{e l}^{n+1}} \\
\mathbf{A}_{n}^{\mathbf{e l}^{n+1}} & \simeq \varepsilon^{\mathbf{e l}^{n+1}} \\
\mathbf{E}_{n}^{\mathbf{e l}^{n+1}} & \simeq \varepsilon^{\mathbf{e l}^{n+1}} \\
\mathbf{E}^{\mathbf{e l}^{n+1}} & \simeq \mathbf{E}^{\mathbf{e l}^{n}}+\mathbf{E}_{n}^{\mathbf{e l}^{n+1}} \\
\mathbf{R}_{0}^{n} & \simeq \mathbf{I} \\
\mathbf{\Sigma}^{n} & \simeq \mathcal{H}: \mathbf{E}_{0}^{\mathbf{e l}^{n}}
\end{aligned}
$$

and let us assume a uniform traction problem with a displacement $x$. Then, it comes, for one element of length $l$ and section $A$, the following deformations

$$
\varepsilon_{n}^{n+1}=\mathbf{F}_{n}^{n+1}-\mathbf{I}=\frac{x^{n+1}-x^{n}}{l}\left(\begin{array}{ccc}
1 & 0 & 0 \\
0 & \frac{2 G-3 k}{6 k+2 G} & 0 \\
0 & 0 & \frac{2 G-3 k}{6 k+2 G}
\end{array}\right)
$$

with $k$ the bulk modulus and $G$ the shear modulus. The stress tensors become

$$
\begin{aligned}
\boldsymbol{\Sigma}^{n} & =\frac{x^{n}}{l}\left(\begin{array}{ccc}
\frac{9 k G}{3 k+G} & 0 & 0 \\
0 & 0 & 0 \\
0 & 0 & 0
\end{array}\right) \\
\boldsymbol{\Sigma}^{n+1} & =\frac{x^{n+1}}{l}\left(\begin{array}{ccc}
\frac{9 k G}{3 k+G} & 0 & 0 \\
0 & 0 & 0 \\
0 & 0 & 0
\end{array}\right)
\end{aligned}
$$


The forces vectors (5) and (32) become (at the first order in $x$ )

$$
\begin{aligned}
& \vec{F}_{i n t}^{n+\frac{1}{2}}=\frac{9 k G A}{[3 k+G] l} \frac{x^{n+1}+x^{n}}{2}\left(\begin{array}{l}
1 \\
0 \\
0
\end{array}\right) \\
& F_{d i s s}^{n+\frac{1}{2}}=\frac{9 k G A}{[3 k+G] l} \chi \frac{x^{n+1}-x^{n}}{2}\left(\begin{array}{l}
1 \\
0 \\
0
\end{array}\right)
\end{aligned}
$$

If, for a translation motion, the kinematic potential (30) is rewritten

$$
D_{K}=\chi \frac{1}{2}\left[\dot{\vec{x}}^{n+1}-\dot{\vec{x}}^{n}\right]^{2}
$$

and if $\omega^{2}=\frac{18 k G}{[3 k+G] \rho l^{2}}$, equations $(23)$ and (25) lead to

$$
\begin{aligned}
& x^{n+1}=x^{n}+\frac{\Delta t}{2}\left[1+\chi \frac{\dot{x}^{n+1}-\dot{x}^{n}}{\dot{x}^{n+1}+\dot{x}^{n}}\right]\left[\dot{x}^{n+1}+\dot{x}^{n}\right] \\
& \dot{x}^{n+1}=\dot{x}^{n}+\frac{\Delta t}{2}\left[\ddot{x}^{n+1}+\ddot{x}^{n}\right] \\
& \ddot{x}^{n+1}=-\ddot{x}^{n}-\omega^{2}\left[1+\chi \frac{x^{n+1}-x^{n}}{x^{n+1}+x^{n}}\right]\left[x^{n+1}+x^{n}\right]
\end{aligned}
$$

This system of equations is equivalent to the one obtained by Armero and Romero ${ }^{15}$ for a spring model, and leads to a spectral matrix with no bifurcation of the eigen values and, for a pulsation equal to the infinity, to a spectral radius

$$
\rho_{\infty}=\frac{1-\chi}{1+\chi}
$$

\section{Blade Loss Simulation}

In this section we present the numerical simulation of a blade loss event in a turbo-engine. We will use the EDMC implicit algorithm presented in the previous section. Contact interactions are simulated with the consistent penalty method proposed by Armero et Petöcz. ${ }^{13,14}$ Let us note that we adapted this method to normal-discontinuous surfaces. ${ }^{22}$ The time step size and the choice of updating the Hessian matrix are computed from automatic criterions, ${ }^{23}$ allowing the implicit simulation to occur in a reduced computational time.

\section{A. Description of the model}

The turbo-engine is modeled with a shaft that has an imposed revolution motion on one extremity. At its other extremity, there is a disk with 24 blades. The shaft, the disk and the blades are part of the rotor, which is in rotation in a stator. The stator is composed from a casing and a bearing.

The rotor has a cyclic symmetry of 15 degrees. Figure 1 . illustrates a 15degree part. The blade is defined from a ruled surface that has two splines for extremities. The two splines are described in Table 1. The blade is made of an alloy (density $\rho=3600 \mathrm{~kg} / \mathrm{m}^{3}$, Young's modulus $Y=88000 \mathrm{~N} / \mathrm{mm}^{2}$, Poisson's coefficient $\nu=0.31$, initial yield stress $\Sigma_{0}=880 \mathrm{~N} / \mathrm{mm}^{2}$ and hardening parameter $\left.h=26700 \mathrm{~N} / \mathrm{mm}^{2}\right)$. The disk and the shaft are composed of another alloy (density $\rho=6300 \mathrm{~kg} / \mathrm{m}^{3}$, Young's modulus $Y=165000 \mathrm{~N} / \mathrm{mm}^{2}$, Poisson's coefficient $\nu=0.31$, initial yield stress $\Sigma_{0}=800 \mathrm{~N} / \mathrm{mm}^{2}$, hardening parameter 
$h=271 \mathrm{~N} / \mathrm{mm}^{2}$ ). The blade is discretized with 99 elements: 11 in length (elements at the head of the blade are $50 \%$ smaller than at the root), 9 elements in height and 1 element on the thickness. The disk has 2 elements on the thickness and 72 elements on the circumference. The shaft has 1 element on its thickness and 11 elements on its length ( 8 for the constant section shaft and 3 for the conical part). The shaft has 72 elements on its circumference. The elements are 8 -node bricks with constant pressure.

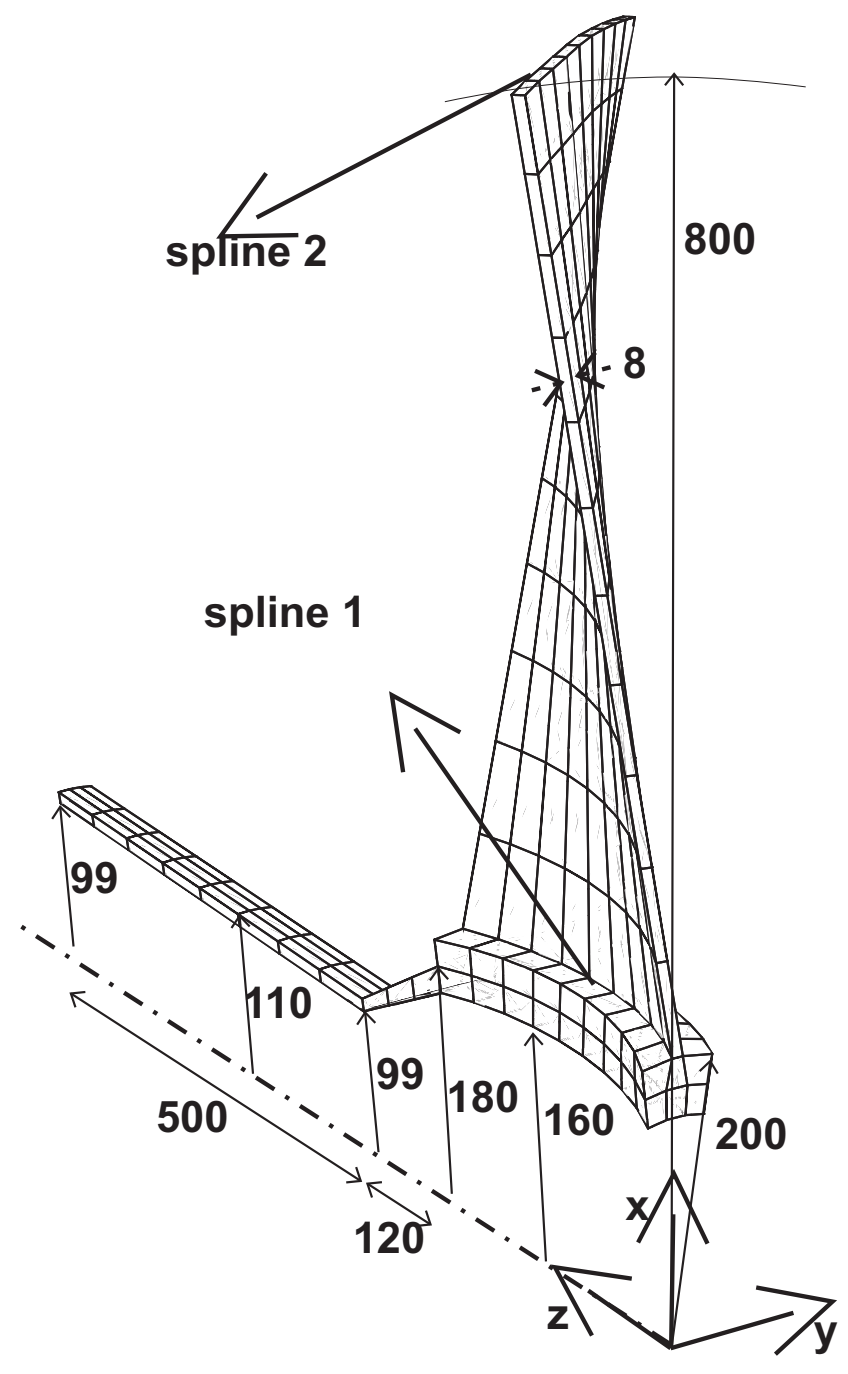

Fig. 1. Rotor model (dimensions in $\mathrm{mm}$ ).

The casing is a cylinder made of an aluminum alloy (density $\rho=2710 \mathrm{~kg} / \mathrm{m}^{3}$, Young's modulus $Y=55200 \mathrm{~N} / \mathrm{mm}^{2}$, Poisson's coefficient $\nu=0.31$, yield stress $\Sigma_{0}=550 \mathrm{~N} / \mathrm{mm}^{2}$ and hardening parameter $h=281 \mathrm{~N} / \mathrm{mm}^{2}$ ). Its geometry is illustrated at Fig. 2.a. The bearing has a conical geometry (Fig. 2.) and is made of an alloy (density $\rho=3600 \mathrm{~kg} / \mathrm{m}^{3}$, Young's modulus $Y=88000 \mathrm{~N} / \mathrm{mm}^{2}$, Poisson's coefficient $\nu=0.31$, yield stress $\Sigma_{0}=550 \mathrm{~N} / \mathrm{mm}^{2}$ and hardening parameter $h=2600 \mathrm{~N} / \mathrm{mm}^{2}$ ). The displacement of the shaft is restrained by the bearing thanks to a central node (Fig. 2.b). There are springs between the central node and the extremity nodes of the bearing and there are springs between the central node and two rows on nodes of the shaft. Each spring has a stiffness of $10^{9} \mathrm{~N} / \mathrm{mm}$. The bearing and the casing have 1 element on the thickness. The casing has 36 elements on the circumference and 8 elements on its length. The bearing has 3 elements on its length and 20 on its circumference. The elements are 8-node bricks with constant pressure. 
Table 1. Coordinates $(\mathrm{mm})$ of the splines defining the blade geometry

\begin{tabular}{ccc}
\hline \hline Point & spline 1 & spline 2 \\
\hline 1 & $\vec{x}=(200 ; 0 ; 0)$ & $\vec{x}=(791.9 ;-113.4 ; 8.9)$ \\
2 & $\vec{x}=(199.3 ; 8.9 ; 19.7)$ & $\vec{x}=(793.9 ;-94.7 ; 28.2)$ \\
3 & $\vec{x}=(198.8 ; 16.3 ; 39.9)$ & $\vec{x}=(795.1 ;-75.9 ; 47.5)$ \\
4 & $\vec{x}=(198.3 ; 22.1 ; 60.7)$ & $\vec{x}=(796.4 ;-57.0 ; 66.7)$ \\
5 & $\vec{x}=(198 ; 26.3 ; 81.9)$ & $\vec{x}=(797.6 ;-38.2 ; 85.9)$ \\
6 & $\vec{x}=(197.9 ; 28.9 ; 103.4)$ & $\vec{x}=(798.8 ;-19.4 ; 105.2)$ \\
7 & $\vec{x}=(197.8 ; 29.7 ; 125)$ & $\vec{x}=(800 . ;-0.6 ; 124.4)$ \\
8 & $\vec{x}=(197.9 ; 28.9 ; 146.6)$ & $\vec{x}=(799.8 ; 18.3 ; 143.7)$ \\
9 & $\vec{x}=(198 ; 26.3 ; 168.0)$ & $\vec{x}=(799.1 ; 37.1 ; 162.9)$ \\
10 & $\vec{x}=(198.3 ; 22.1 ; 189.2)$ & $\vec{x}=(798.0 ; 55.7 ; 182.3)$ \\
11 & $\vec{x}=(198.8 ; 16.3 ; 210.1)$ & $\vec{x}=(796.5 ; 74.3 ; 201.8)$ \\
12 & $\vec{x}=(199.3 ; 8.9 ; 230.3)$ & $\vec{x}=(794.6 ; 92.6 ; 221.4)$ \\
13 & $\vec{x}=(200 ; 0 ; 0.25)$ & $\vec{x}=(792.3 ; 110.7 ; 241.3)$ \\
\hline \hline
\end{tabular}

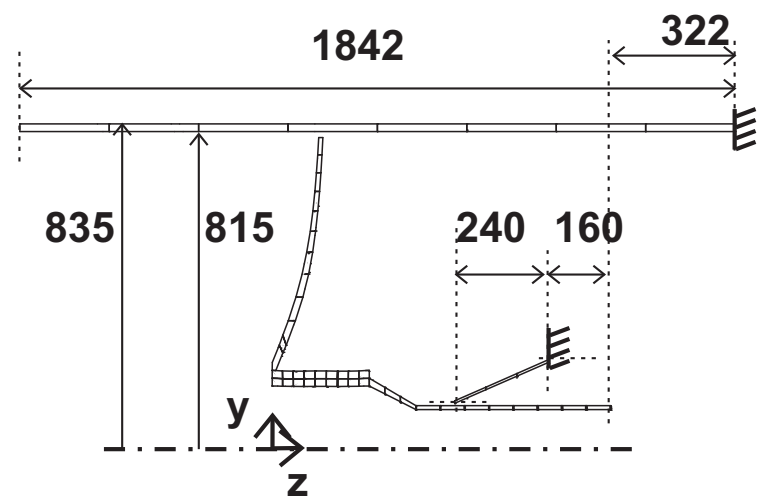

a. casing

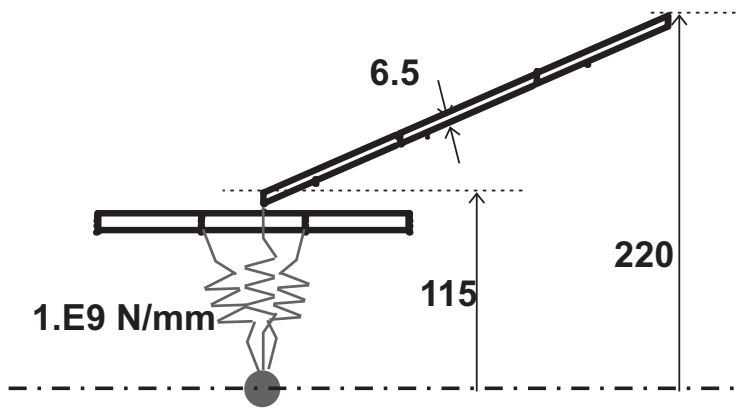

b. bearing

Fig. 2. Stator model (dimensions in $\mathrm{mm}$ ).

\section{B. Initial conditions}

At time $t=0 s$, the initial configuration of the rotor is computed for a rotation velocity of $4775 \mathrm{rpm}$. This initial configuration is computed with a NewtonRaphson scheme where the external forces are the analytical inertial forces computed from the nodes position and from the imposed rotation velocity. The von Mises stresses resulting from this uniform rotation velocity are illustrated at Fig. 3. The blade pointed by an arrow is independent from the disk. To evaluate the initial configuration, it is linked to the disk thanks to an adhesion law (normal penalty $k_{p}=10^{9}$, tangential penalty $k_{T}=10^{8}$ ). After the initial configuration is evaluated, this link is removed and the free blade interacts with the other blades and with the casing. The interaction between the blades and the casing is simulated with a Coulomb friction law (normal penalty $k_{p}=10^{9}$ tangential penalty $k_{T}=10^{7}$, friction coefficient $\mu_{c}=0.1$ ). The interaction between the free blade and the other blades is simulated with the same law. Contact interactions between attached blades are simulated with a frictionless law (normal penalty $k_{p}=10^{9}$ ). 


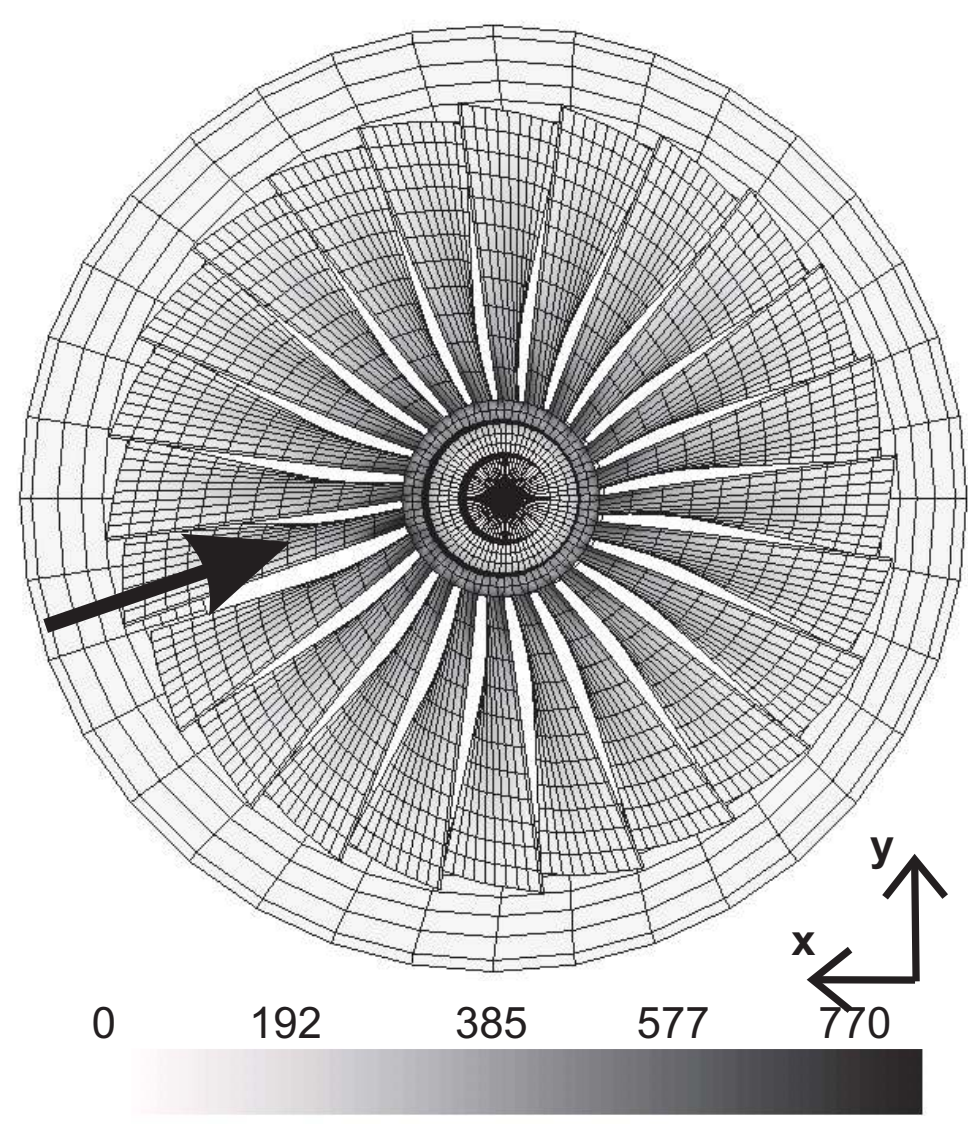

Fig. 3. Initial von Mises stress $\left(\mathrm{N} / \mathrm{mm}^{2}\right)$ of the rotor (front view).

\section{First revolution}

In this section we analyze the first revolution of the rotor after the blade loss. We use the EDMC (first order accurate) algorithm with a spectral radius equal to $\rho_{\infty}=0.8$. The time step size is computed from an automatic criterion ${ }^{23}$ and with an accuracy of $10^{-4}$ on the integration error. ${ }^{23}$ Each time step is computed with a Newton-Raphson scheme (Tolerance $=10^{-5}$ ) enhanced by a line-search system $^{24}$ (Tolerance $\left.=10^{-3}\right)$.

Figure 4.a illustrates the configuration after a quarter of revolution. The free blade interact with the first next (attached) blade. Figure 4.b illustrates the configuration after half a revolution. The free blade remains between the linked blades and the casing. Due to the friction law, the attached blades bend. The head of the free blade enters into contact with the fifth linked blade. Figure 4.c illustrates the deformation after three quarters of a revolution. The head of the free blade has led the fifth blade to bend significantly, and the free blade is pushed towards the rear of the casing. Figure 4.d illustrates the results after a revolution. The free blade was pushed away from the disk so that the remaining interactions only occur between the linked blades and the casing. 


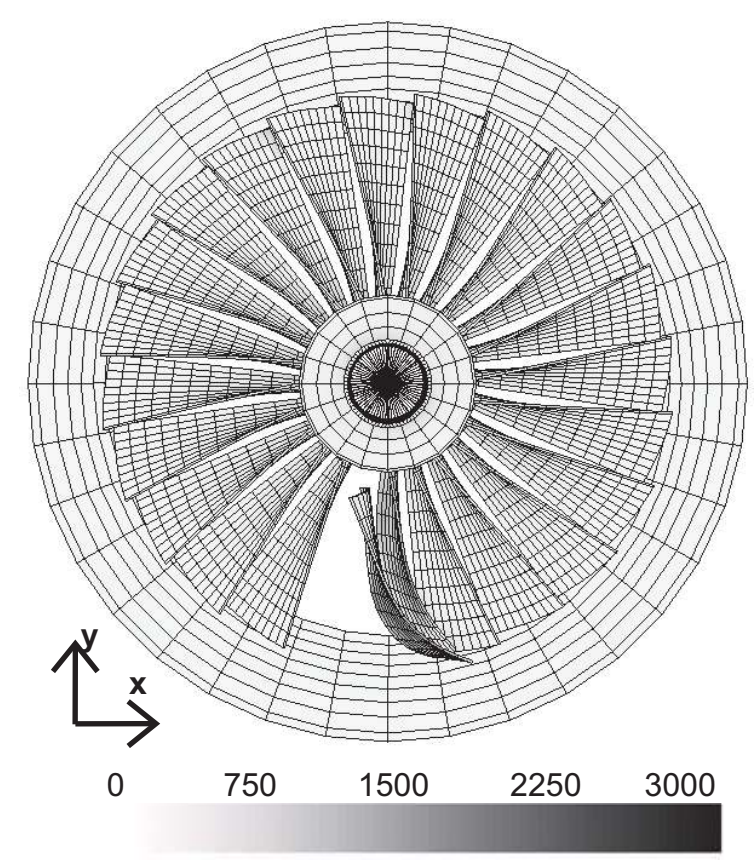

a. 1/4 round

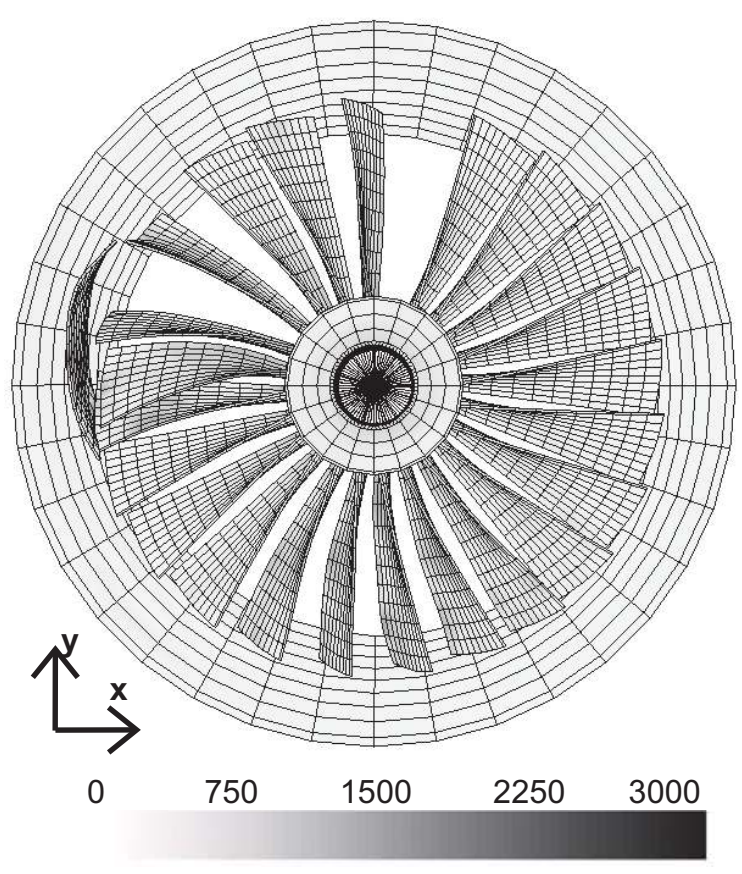

c. $3 / 4$ round

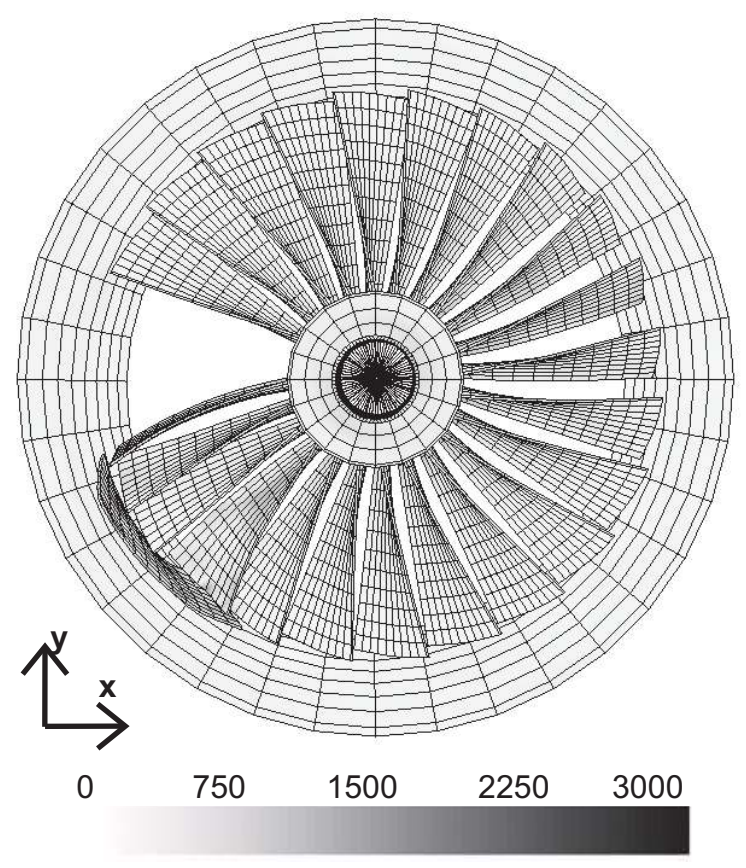

b. $1 / 2$ round

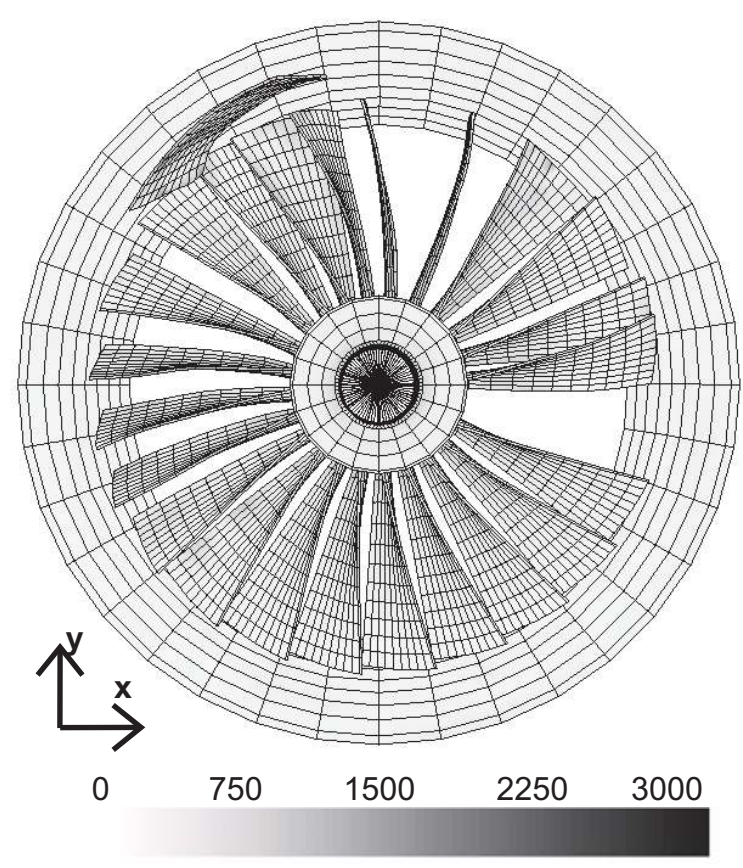

d. 1 round

Fig. 4. Configuration and von Mises stress distribution $\left(N / m m^{2}\right)$ during the first round (rear view).

The total force on the bearing is illustrated at Fig. 5.a. It appears that this force is linear during the first instants, when the bearing reacts to the presence of an unbalanced shaft. But when the free blade interact with both the linked blades and the casing, the force starts oscillating. The time evolution of the force on the casing (Fig. 5.b) results from the interaction of the blades on the casing and the force oscillates during the whole simulation. 


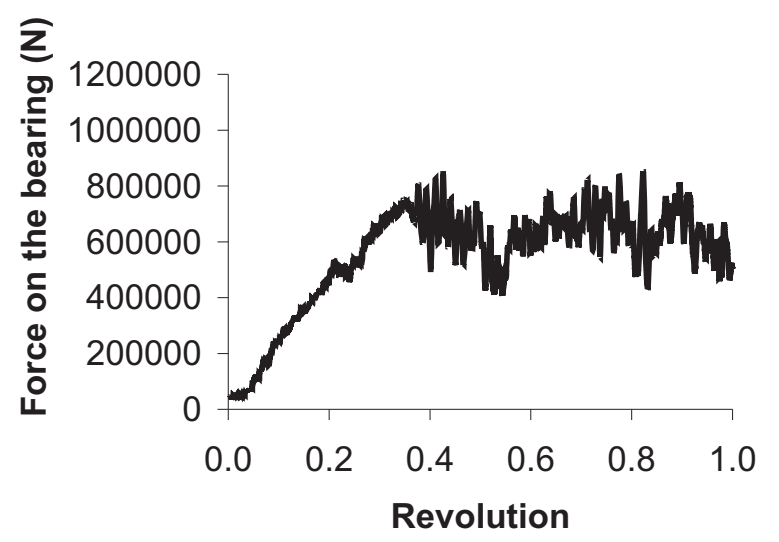

a. Force on the bearing

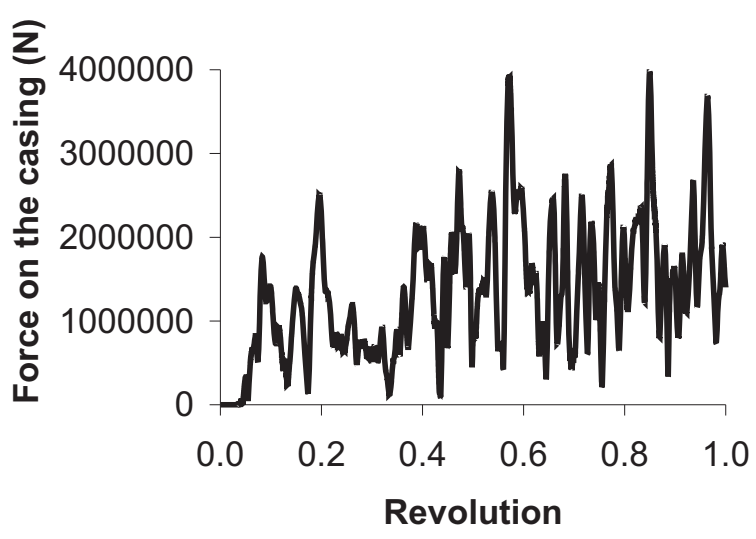

b. Force on the casing

Fig. 5. Time evolution of forces on the stator.

\section{Conclusions}

In this paper we have presented a new dissipative implicit algorithm, for hypoelastic models, that is consistent in the non-linear range. This algorithm is first order accurate, conserves the linear and the angular momenta, and we demonstrated that the numerical dissipation always remains positive in the nonlinear range.

Thanks to the numerical dissipation and this consistency, we were able to presents a complex numerical simulation of blade loss. Therefore we conclude that this new kind of energy-momentum consistent algorithm in the non-linear range is able to simulate complex dynamics phenomena that were usually simulated with explicit schemes. The advantage of the implicit simulations (by opposition to the explicit simulations) is the proof of the stability, and therefore of the accuracy, in the non-linear range.

\section{Acknowledgments}

L. Noels and L. Stainier thank the Belgian National Fund for Scientific Research (F.N.R.S.) for its support.

\section{References}

${ }^{1}$ Newmark, N., "A method of computation for structural dynamics," Journal of the Engineering Mechanics Division ASCE, Vol. 85, No. EM3, 1959, pp. 67-94.

${ }^{2}$ Belytschko, T. and Schoeberle, D., "On the unconditional stability of an implicit algorithm for non-linear structural dynamics," Journal of Applied Mechanics, Vol. 42, 1975, pp. 865-869.

${ }^{3}$ Hughes, T., "A note on the stability of Newmark's algorithm in nonlinear structural dynamics," International Journal of Numerical Methods in Engineering, Vol. 11, No. 2, 1977, pp. 383-386.

${ }^{4}$ Hughes, T., "Stability, convergence and growth and decay of energy of the average acceleration method in nonlinear structural dynamics," Computers $\&$ Structures, Vol. 6, 1976, pp. 313-324.

${ }^{5}$ Hughes, T., Caughey, T., and Liu, W., "Finite-element method for non-linear elastodynamics which conserve energy," Journal of Applied Mechanics, Vol. 45, june 1978, pp. 366-370.

${ }^{6}$ Chung, J. and Hulbert, G., "A time integration algorithms for structural dynamics with improved numerical dissipations: the generalized- $\alpha$ method," Journal of Applied Mechanics, Vol. 60, 1993, pp. 371-375. 
${ }^{7}$ Erlicher, S., Bonaventura, L., and Bursi, O., "The analysis of the $\alpha$-generalized method for non-linear dynamic problems," Computational Mehanics, Vol. 28, 2002, pp. 83-104.

${ }^{8}$ Simo, J. and Tarnow, N., "The discrete energy-momentum method. Conserving algorithms for nonlinear elastodynamics," ZAMP, Vol. 43, 1992, pp. 757-792.

${ }^{9}$ Gonzalez, O., "Exact energy and momentum conserving algorithms for general models in nonlinear elasticity," Computer Methods in Applied Mechanics and Engineering, Vol. 190, 2000, pp. 1763-1783.

${ }^{10}$ Meng, X. and Laursen, T., "Energy consistent algorithms for dynamic finite deformation plasticity," Computer Methods in Applied Mechanics and Engineering, Vol. 191, 2001, pp. 1639-1675.

${ }^{11}$ Noels, L., Stainier, L., and Ponthot, J.-P., "Energy-momentum conserving algorithm for non-linear hypoelastic constitutive models," International Journal of $\mathrm{Nu}$ merical Methods in Engineering, Vol. 59, 2004, pp. 83-114.

${ }^{12}$ Noels, L., Stainier, L., and Ponthot, J.-P., "On the use of large time steps with an energy-momentum conserving algorithm for non-linear hypoelastic constitutive models," International Journal of Solids and Structures, Vol. 41, 2004, pp. 663-693.

${ }^{13}$ Armero, F. and Petcz, E., "Formulation and analysis of conserving algorithms for frictionless dynamic contact/impact problems," Computer Methods in Applied Mechanics and Engineering, Vol. 158, 1998, pp. 269-300.

${ }^{14}$ Armero, F. and Petcz, E., "A new dissipative time-stepping algorithm for frictional contact problems: formulation and analysis," Computer Methods in Applied Mechanics and Engineering, Vol. 179, 1999, pp. 151-178.

${ }^{15}$ Armero, F. and Romero, I., "On the formulation of high-frequency dissipative time-stepping algorithms for non-linear dynamics. Part I: low-order methods for two model problems and nonlinear elastodynamics," Computer Methods in Applied Mechanics and Engineering, Vol. 190, 2001, pp. 2603-2649.

${ }^{16}$ Armero, F. and Romero, I., "On the formulation of high-frequency dissipative time-stepping algorithms for non-linear dynamics. Part II: second-order methods," Computer Methods in Applied Mechanics and Engineering, Vol. 190, 2001, pp. 67836824

${ }^{7}$ Nagtegaal, J. and Veldpaus, F., "On the implementation of finite strain plasticity equations in a numerical model," Numerical Analysis of Forming Processes, Pittman et al. (Eds) John Wiley and sons, 1984, pp. 351-371.

${ }^{18}$ Ponthot, J.-P., "Unified stress update algorithms for the numerical simulation of large deformation elasto-plastic and elasto-viscoplastic processes," International Journal of Plasticity, Vol. 18, 2002, pp. 91-126.

${ }^{19}$ Wilkins, M., "Calculation of elastoplastic flows," Methods of Computational Physics, Vol. 3, 1964, pp. 211-263.

${ }^{20}$ Maenchen, M. and Sack, S., "The tensor code," Methods of Computational Physics, Vol. 3, 1964, pp. 387-400.

${ }^{21}$ Noels, L., Stainier, L., and Ponthot, J.-P., "Simulation of complex impact problems with implicit time algorithm. Application to a blade-loss problem," International Journal of Impact Engineering, in preparation.

${ }^{22}$ Noels, L., Stainier, L., and Ponthot, J.-P., "Simulation of crashworthiness problems with new improvement in implicit time integration of non linear dynamical systems," International Journal of Impact Engineering, in preparation.

${ }^{23}$ Noels, L., Stainier, L., and Ponthot, J.-P., "Self-adapting time integration management in crash-worthiness and sheet metal forming computations," International Journal of Vehicle Design, Vol. 30, No. 2, 2002, pp. 1-48.

${ }^{24}$ Crisfield, M., Non-linear finite element analysis of solids and structures, Vol. 1, John Wiley and Sons, 2001. 